\title{
OPTIMAL STRATEGIES FOR BILEVEL DYNAMIC PROBLEMS
}

\author{
JANE J. YE
}

DMS-645-IR

August 1993 
Lrent. te

\title{
OPTIMAL STRATEGIES FOR BILEVEL DYNAMIC PROBLEMS*
}

\author{
Jane J. Ye
}

August 31, 1993

\begin{abstract}
In this paper we study the bilevel dynamic problem, which is a heierarchy of two dynamic optimization problems where the constraint region of the upper level problem is determined implicitly by the solution to the lower level optimal control problem. To obtain optimality conditions, we reformulate the bilevel dynamic problem as a single level optimal control problem which involves the value function of the lower level problem. Sensitivity analysis of the lower level problem with repect to the perturbation in the upper level decision variable is given and first order necessary optimality conditions are derived by using nonsmooth analysis.
\end{abstract}

Key words: necessary conditions, bilevel optimal control problems, sensitivity analysis, nonsmooth analysis, value funtion.

AMS(MOS) subject classification: 90D65, 49K40

Abbreviated Title: Bilevel Dynamic Problems

* This work was supported by NSERC under grant WFA 0123160

${ }^{\dagger}$ Department of Mathematics and Statistics, University of Victoria, Victoria, B.C., Canada V8W $3 \mathrm{P} 4$. 


\section{Introduction}

Let us consider a two-level hierachical system where two decision makers try to find best decisions with respect to certain, but generally different goals. Moreover, assume that these decision makers cannot act independently of each other but only according to a certain hierarchy, whereby the optimal strategy chosen by the lower level (hereafter the "follower") depends on the strategy selected by the upper level (hereafter the "leader"). On the other hand, let the objective function of the leader depend not only on its own decision but also on the reaction of the follower. Then while having the first chocie, the leader is able to evaluate the true value of his own selection only after knowing the follower's possible reactions. Assume that the game is cooperative, i.e., if the follower's problem has several optimal decisions for a given leader's decision, then the follower allows the leader to choose which of them is actually used. Thus the leader will choose his optimal decision among all decisions available and the follower's optimal decision to minimize his objective. In particular, we consider a hierachical dynamical systems where the state $x(t) \in R^{d}$ is influenced by the decisions of both leader and follower $u(\cdot)$ and $v(\cdot)$. The state $x(t) \in R^{d}$ is described by

$$
\begin{aligned}
\dot{x}(t) & =\phi(t, x(t), u(t), v(t)) \quad \text { a.e. } t \in\left[t_{0}, t_{1}\right] \\
x\left(t_{0}\right) & =x_{0},
\end{aligned}
$$

where $u(t) \in R^{n}, v(t) \in V(t) \subset R^{m}$. In mathematical terms, given any control function $u(\cdot)$ selected by the leader, the follower faces the ordinary (single level) optimal control problem involving a parameter $u$ :

$$
\begin{aligned}
P_{2}(u) \quad \min \quad & J_{2}(x, u, v)=\int_{t_{0}}^{t_{1}} G(t, x(t), u(t), v(t)) d t+g\left(x\left(t_{1}\right)\right) \\
\text { s.t. } \quad & \dot{x}(t)=\phi(t, x(t), u(t), v(t)) \quad \text { a.e. } \\
& x\left(t_{0}\right)=x_{0}, \\
& v(t) \in V(t) \quad \text { a.e., }
\end{aligned}
$$

while the leader faces the bilevel dynamic problem:

$$
\begin{array}{lll}
P_{1} & \min & J_{1}(x, u, v)=\int_{t_{0}}^{t_{1}} F(t, x(t), u(t), v(t)) d t+f\left(x\left(t_{1}\right)\right) \\
& \text { over } \quad u \in L^{2}\left(\left[t_{0}, t_{1}\right], R^{n}\right) \text { and all solutions }(x, v) \text { of } P_{2}(u) .
\end{array}
$$

The bilevel static problem where both leader and follower's decisions are vectors instead of control functions was first introduced by von Stackelberg [10] for an economic model. The bilevel dynamic problems were first considered by Chen and Cruz 
in [2]. Most of the bilevel (static or dynamic) problems are attacked by reducing the bilevel problem to a single level problem with the first order necessary conditions for the lower level problem as additional constriants ( $c f$. Bard and Falk [1] and Zhang [13] [14] for bilevel static problems, Chen and Cruz [2] and Zhang [13] for bilevel dynamic problems). The reduction is equivalent if and only if the lower level optimal control problem is convex since in this case the first order necessary condition is also sufficient. Apart from the strong convexity assumption, the resulting optimality conditions of the above approach involve second order derivatives and a larger system since the reduced problem minimizes over the set of original decesion variables as well as the set of multipliers of the lower level problem.

To our knowledge, there is no optimality conditions for a general bilevel dynamic problem to date. The necessary condition obtained by Chen and Cruz in [2] holds in the case where the Pontryagin's maximum principle for the lower level optimal control problem is sufficient for optimality and no bounds are allowed for the control functions. The necessary condition was stated in a normal form (i.e. the mulitiplier for the objective function of the upper level problem is 1) which only holds when the reduced single level optimal control problem is calm (see [4] for definition). The necessary condition obtained by Zhang in [13] is only for a bilevel dynamic problem in which the dynamic is linear in the state and control variables and requires convexity assumptions on the objective function of the lower level problem. The purpose of this paper is to provide first order necessary conditions for problem $P_{1}$ under very general assumptions (in particular, without convexity assumptions and with bounds on the control functions on the lower level problem).

Define the value function of the lower level optimal control problem as an extendedvalued functional $V(u): L^{2}\left(\left[t_{0}, t_{1}\right], R^{n}\right) \rightarrow \bar{R}$ defined by

$$
V(u):=\inf \left\{\begin{array}{cl}
\int_{t_{0}}^{t_{1}} G(t, x(t), u(t), v(t)) d t+g\left(x\left(t_{1}\right)\right): & \dot{x}(t)=\phi(t, x(t), u(t), v(t)) \text { a.e. } \\
& v(t) \in V(t) \quad \text { a.e. } \\
& x\left(t_{0}\right)=x_{0}
\end{array}\right\}
$$

where $\vec{R}:=R \cup\{-\infty\} \cup\{+\infty\}$ is the extended real line and inf $\emptyset=+\infty$ by convention. Our approach is to reformulate $P_{1}$ as the following single level optimal control problem:

$$
\begin{array}{rll}
\tilde{P}_{1} \quad \min & J_{1}(u, v)=\int_{t_{0}}^{t_{1}} F(t, x(t), u(t), v(t)) d t+f\left(x\left(t_{1}\right)\right) \\
\text { s.t. } & \dot{x}(t)=\phi(t, x(t), u(t), v(t)) \quad \text { a.e. }
\end{array}
$$




$$
\begin{aligned}
& x\left(t_{0}\right)=x_{0} \\
& u(\cdot) \in L^{2}\left(\left[t_{0}, t_{1}\right], R^{n}\right), v(t) \in V(t) \quad \text { a.e. } \\
& V(u) \geq \int_{t_{0}}^{t_{1}} G(t, x(t), u(t), v(t)) d t+g\left(x\left(t_{1}\right)\right) .
\end{aligned}
$$

The above problem is obviously equivalent to the original bilevel dynamic problem $P_{1}$ and is a nonstandard optimal control problem since the constraints involve a functional defined by the value function $V(u)$ of the lower level optimal control problem. In general $V(u)$ is not an explicit function of the problem data and is nonsmooth even in the case where all problem data are smooth functions. To derive a necessary condition for optimality for problem $P_{1}$, one needs to study the Lipschitz continuity and the generalized derivatives of the value function $V(u)$ and develop a necessary optimality condition for the nonstandard optimal control problem with functional constraints. Recent development in nonsmooth analysis allow us to study the Lipschitz continuity and generalized derivative of the value function with respect to infinite-dimensional perturbation $V(u)$. We then reformulate the nonstandard optimal control problem as an infinite dimensional optimization problem and use a result due to Ioffe [8] to derive a necessary optimality condition for the nonstandard optimal control with functional constraints.

The approach of reducing a bilevel problem to a single level problem was first used by Ye and Zhu [12] to derive first order necessary conditions for the static bilevel optimization problem. In Ye [11], a bilevel dynamic optimization problem where the lower level is an optimal control problem while the upper level decision variable is a vector is considered. Although the bilevel dynamic optimization problem considered in [11] is a special case of the problem we study in this paper, it deserves a special attention since it reduces to a single level optimal control problem with end point contraints involving a value function which is a function of the upper level decision vector. Different techique from the one we use for this paper was used and hence a necessary optimality condition with stronger results and more general assumptions than otherwise derived from being a special case of the bilevel dynamic problem studied in this paper has been derived.

The following basic assumptions are in force throughout this paper:

(A1) $V(t):\left[t_{0}, t_{1}\right] \rightarrow R^{m}$ is nonempty compact valued set-valued map. The graph of $V(t)$ (i.e., the set $\left\{(s, r): s \in\left[t_{0}, t_{1}\right], r \in V(s)\right\}$ ), denoted by $\operatorname{Gr} V$, is $\mathcal{L} \times$ $\mathcal{B}$ measurable, where $\mathcal{L} \times \mathcal{B}$ denotes the $\sigma$-algebra of subsets of $\left[t_{0}, t_{1}\right] \times \mathbb{R}^{m}$ 
generated by product sets $M \times N$ where $M$ is a Lebesgue measurable subset of $\left[t_{0}, t_{1}\right]$ and $N$ is a Borel subset of $\mathbb{R}^{m}$;

(A2) The function $F(t, x, u, v):\left[t_{0}, t_{1}\right] \times R^{d} \times R^{n} \times R^{m} \rightarrow R$ is $\mathcal{L} \times \mathcal{B}$ measurable in $(t, v)$ and continuously differentiable in $x$ and $u$. The functions $\phi(t, x, u, v)$ : $\left[t_{0}, t_{1}\right] \times R^{d} \times R^{n} \times R^{m} \rightarrow R^{d}, G(t, x, u, v):\left[t_{0}, t_{1}\right] \times R^{d} \times R^{n} \times R^{m} \rightarrow R$ are measurable in $t$, continuously differentiable in $x$ an $u$ and lower semicontinous in $v$.

(A3) There exists an integrable function $\psi:\left[t_{0}, t_{1}\right] \rightarrow R$ such that

$$
\left|\nabla_{(x, u)} F\right|+\left|\nabla_{(x, u)} G\right|+\left|\nabla_{(x, u)} \phi\right| \leq \psi(t) .
$$

(A4) The function $f(x): \mathbb{R}^{d} \rightarrow R$ is locally Lipschitz continuous and the function $g(x): R^{d} \rightarrow R$ is Lipschitz continuous of rank $L_{g}$.

(A5) For any $u \in L^{2}\left(\left[t_{0}, t_{1}\right], R^{n}\right), P_{2}(u)$ has an admissible pair (whose definition is given below).

A control function for $P_{2}(u)$ is a (Lebesgue) measurable selection $v(\cdot)$ for $V(\cdot)$; that is a measurable function satisfying $v(t) \in V(t)$ a.e. $t \in\left[t_{0}, t_{1}\right]$. An arc is an absolutely continuous function. An admissible pair for $P_{2}(u)$ is a pair of functions $(x(\cdot), v(\cdot))$ on $\left[t_{0}, t_{1}\right]$ of which $v(\cdot)$ is a control function for $P_{2}(u)$ and $x(\cdot):\left[t_{0}, t_{1}\right] \rightarrow R^{d}$ is an arc which satisfies the differential equation $\dot{x}(t)=\phi(t, x(t), u(t), v(t))$ a.e., together with the initial condition $x\left(t_{0}\right)=x_{0}$. The first and the second components of an admissible pair are called admissible trajectory and admissible control respectively. A solution to problem $P_{2}(u)$ is an admissible pair for $P_{2}(u)$ which minimizes the value of the cost functional $J_{2}(x, u, v)$ over all admissible pairs for $P_{2}(u)$. An admissible strategy for $P_{1}$ includes $u \in L^{2}\left(\left[t_{0}, t_{1}\right], R\right)$ and an optimal control $v$ for $P_{2}(u)$. The strategy $(u, v)$ and the corresponding trajectory $x$ are optimal for the bilevel dynamic problem $P_{1}$ if $(x, u, v)$ minimizes the value of the cost functional $J_{1}(x, u, v)$ among all admissible strategies and the corresponding trajectories for $P_{1}$.

The plan of the paper is as follows: In section 2 , we study generalized differentiability of the value function $V(u)$. The necessary condition for optimality is given in section 3 . 


\section{Differentiability of the value function}

Let $X$ be a Hilbert space. Consider a lower semicontinuous functional $\phi: X \rightarrow$ $\mathbb{R} \cup\{+\infty\}$ and a point $\bar{x} \in X$ where $\phi$ is finite. A vector $\zeta \in X$ is called a proximal subgradient of $\phi(\cdot)$ at $\bar{x}$ provided that there exist $M>0, \delta>0$ such that

$$
\left\langle\zeta, x^{\prime}-\bar{x}\right\rangle \leq \phi\left(x^{\prime}\right)-\phi(\bar{x})+M\left\|x^{\prime}-\bar{x}\right\|^{2} \quad x^{\prime} \in \bar{x}+\delta B .
$$

The set of all proximal subgradients of $\phi(\cdot)$ at $\bar{x}$ is denoted $\partial^{\pi} \phi(\bar{x})$. A limiting subgradient of $\phi$ at $\bar{x}$ is the set

$$
\hat{\partial} \phi(\bar{x}):=\left\{\text { weak }^{*} \lim _{k \rightarrow \infty} \zeta_{k}: \zeta_{k} \in \partial^{\pi} \phi\left(x_{k}\right), x_{k} \rightarrow \bar{x}, \phi\left(x_{k}\right) \rightarrow \phi(\bar{x})\right\} .
$$

The limiting subgradient is a smaller object than the Clarke generalized gradient (see Clarke [3] for definition). In fact, if $\phi$ is Lipschitz continuous near $x$, we have $\partial \phi(x)=\operatorname{clco} \hat{\partial} \phi(x)$, where $\partial$ and clcoA denote the Clarke generalized gradient and closed convex hull of set A respectively. For the definition and the precise relation between the limiting subgradient and the Clarke generalized gradient, the reader is referred to Clarke [4] and Rockafellar [9] for more details.

The following result concerning about the compactness of trajectories to a differential inclusion is slightly different from [Theorem 3.1.7. of Clarke [3]] and will be used repeatively. We omit the proof here since it can be proved similarly as [Theorem 3.1.7. of Clarke [3]].

Proposition 2.1 Let $\Gamma$ be set-valued map defined on $\left[t_{0}, t_{1}\right] \times R^{d} \times R^{n}$. We suppose that $\Gamma$ is integrably bounded (i.e., there exists an integrable function $k(t)$ such that $|v| \leq k(t)$ for all $v \in \Gamma(t, x, u)$ ) and that $\Gamma$ is nonempty, compact and convex. We suppose that for every $(t, x, u) \in\left[t_{0}, t_{1}\right] \times R^{d} \times R^{n}$, the set-valued map $t^{\prime} \rightarrow \Gamma\left(t^{\prime}, x, u\right)$ is measurable and for all $\left[t_{0}, t_{1}\right] \times R^{d} \times R^{n}$, the set-valued map $\left(x^{\prime}, u^{\prime}\right) \rightarrow \Gamma\left(t, x^{\prime}, u^{\prime}\right)$ is uppersemicontinuous. Let $\Gamma$ be $\mathcal{L} \times \mathcal{B}$ measurable where $\mathcal{L} \times \mathcal{B}$ denotes the $\sigma$-algebra of subsets of $\left[t_{0}, t_{1}\right] \times R^{d} \times R^{\text {n }}$ generated by product sets $\bar{M} \times \bar{N}$ where $\bar{M}$ is a Lebesque measurable subset of $\left[t_{0}, t_{1}\right]$ and $N$ is a Boreal subset of $R^{d} \times R^{n}$.

Let $\left\{x_{i}\right\}$ be a sequence of arcs on $\left[t_{0}, t_{1}\right]$ and $\left\{\zeta_{i}\right\}$ be a sequence of functions in $L^{2}\left(\left[t_{0}, t_{1}\right], R^{n}\right)$ satisfying:

(i) $\left(\dot{x}_{i}(t), \zeta_{i}(t)\right) \in \Gamma\left(t, x_{i}(t), u_{i}(t)\right) \quad$ a.e. $t \in\left[t_{0}, t_{1}\right]$

(ii) $\zeta_{i} \rightarrow \zeta$ in $L^{2}$ 
(iii) $u_{i} \rightarrow u$ in $L^{2}$

(iv) $\left\{x_{i}\left(t_{0}\right)\right\}$ is bounded.

Then there exists a subsequence of $\left\{x_{i}\right\}$ which converges uniformly to an arc $x$ such that

$$
(\dot{x}(t), \zeta(t)) \in \Gamma(t, x(t), u(t)) \quad \text { a.e. } t \in\left[t_{0}, t_{1}\right] .
$$

To discuss generalized differentiability of the value function $V(u)$, we will need the following assumptions:

(A6) There exists $k(t) \in L^{2}\left(\left[t_{0}, t_{1}\right], R\right)$ such that

$$
|\phi|+\left|\nabla_{x} \phi\right|+|G|+\left|\nabla_{x} G\right|+\left|\nabla_{u} G\right| \leq k(t) .
$$

(A7) For any $(t, x, u) \in\left[t_{0}, t_{1}\right] \times \mathbb{R}^{d} \times R^{n}$, the set

$$
\{(\phi(t, x, u, v), G(t, x, u, v)+r): v \in V(t), r \geq 0\}
$$

is convex.

(A8) The Jacobi matrix $\nabla_{u} \phi$ is invertible and

$$
\left|\left(\nabla_{u} \phi\right)^{-1}\right|+\left|\nabla_{u} \phi\right| \leq M
$$

where $M>0$ is a constant.

In [5], Clarke studied the generalized differentiablity of the value function of an optimal control problem with an additive infinite-dimensional perturbation in the dynamic and pointed out that the extension of the theory to infinite-dimensional perturbations occurring in as general a fashion as the finite-dimensional ones studied by Clarke and Loewen [6] is problematic. Our assumptions allow the nonadditive perturbations both in dynamic and objective functions with restrictions in the perturbation structure (see Asssumption (Â8)). Our result is not strictly more general than Clarke [5] since we can only deal with an optimal control problem without endpoint constraints while [5] has the result for the optimal control problem with endpoint constraints. However, we choose to study the problem without endpoint contraints but with nonadditive perturbations in both dynamic and objective functions instead of the problem with endpoint constraints but with only additive perturbation in the dynamic since the latter is too restrictive for the bilevel dynamic problem where the 
perturbation is the control function of the upper level problem which will usually occur in both dynamic and objective function of the lower level optimal control problem and will usually occur nonadditively. As in the ordinary optimal control problem, the bilevel dynamic problems without endpoint constraints are basic problems to study. Most of the papers in the literature also study the problem without endpoint constraints.

Let Hamiltonian for $P_{2}(u)$ be the function defined by

$$
H_{2}\left(t, x, u, p_{2}\right):=\sup \left\{p_{2} \cdot \phi(t, x, u, v)-G(t, x, u, v): v \in V(t)\right\}
$$

and $Y_{u}$ be the set of all optimal trajectory $x$ to problem $P_{2}(u)$.

The following result gives the Lipschitz continuity of the value function and characterizes the generalized derivatives of the value function.

Theorem 2.1 Suppose assumptions (A1)-(A8) hold. Then $V$ is Lipschitz continuous near $u$ and one has

$$
\begin{aligned}
& \partial V(u) \subset \operatorname{clco} \cup_{x \in Y_{u}}\left\{\zeta: \exists \operatorname{arc} p_{2} \text { s.t. }\left(-\dot{p_{2}}, \zeta, \dot{x}\right) \in \partial H_{2}\left(t, x, u, p_{2}\right)\right. \text { a.e. } \\
& \left.-p_{2}\left(t_{1}\right) \in \hat{\partial} g\left(x\left(t_{1}\right)\right)\right\},
\end{aligned}
$$

where $\partial \mathrm{H}_{2}$ denotes the Clarke generalized gradient respect to $\left(x, u, p_{2}\right)$.

Before proving Theorem 2.1, we first give the following result:

Lemma 2.1 Let $u_{i}$ be a sequence converging (in $\left.L^{2}\right)$ to $u$ and let $\left(x_{i}, v_{i}\right)$ be an admissible pair for $P_{2}\left(u_{i}\right)$. Then there exists a subsequence of $\left\{x_{i}\right\}$ converging uniformly to an $\operatorname{arc} x$ and a control $v$ with $(x, v)$ being an admissible pair for $P_{2}(u)$ such that

$$
J_{2}(x, u, v) \leq \liminf J_{2}\left(x_{i}, u_{i}, v_{i}\right) \text {. }
$$

Proof. Let

$$
\dot{y}_{i}(t):=G\left(t, x_{i}(t), u_{i}(t), v_{i}(t)\right)
$$

Then

$$
\left(\dot{x}_{i}(t), \dot{y}_{i}(t)\right) \in \Gamma\left(t, x_{i}(t), y_{i}(t), u_{i}(t)\right)
$$

where

$$
\Gamma(t, x, y, u):=\{(\phi(t, x, u, v), r): G(t, x, u, v) \leq r \leq k(t)+1, v \in V(t)\}
$$


The proof can be reduced to an application of Proposition 2.1 by studying differential inclusion (1). The essential fact in the reduction is Fillipov's lemma: an (extended) arc $(x, y)$ satisfies the differential inclusion iff there is a control function $v$ for $x$ such that $(x, v)$ is feasible for $P_{2}(u)$ and $y$ satisfies $G(t, x, u, v) \leq \dot{y} \leq k(t)+1$.

We now turn to the proof of the theorem. By (A5), $P_{2}(u)$ has an admissible pair. So $V(u)$ is finite. By Lemma $2.1, V$ is (strongly) lower semicontinuous.

Step 1. Let $u \in L^{2}\left(\left[t_{0}, t_{1}\right], R^{n}\right)$ and $\zeta \in \partial^{\pi} V(u)$. Let $(x, v)$ be a solution of $P_{2}(u)$ which exists by virtue of Lemma 2.1. Then by definition, for some $M>0$ and for all $u^{\prime}$ near $u$ (in the $L^{2}$ norm), we have

$$
\begin{aligned}
V\left(u^{\prime}\right)-\left\langle\zeta, u^{\prime}\right\rangle+M \| u^{\prime}- & u \|_{2}^{2} \geq V(u)-\langle\zeta, u\rangle \\
& =\int_{t_{0}}^{t_{1}} G(t, x(t), u(t), v(t)) d t+g\left(x\left(t_{1}\right)\right)-\int_{t_{0}}^{t_{1}}\langle\zeta(t), u(t)\rangle d t .
\end{aligned}
$$

Let $\left(x^{\prime}, v^{\prime}\right)$ be an admissible pair for $P_{2}\left(u^{\prime}\right)$. Then

$$
\begin{gathered}
\int_{t_{0}}^{t_{1}} G\left(t, x^{\prime}(t), u^{\prime}(t), v^{\prime}(t)\right) d t+g\left(x^{\prime}\left(t_{1}\right)\right)-\int_{t_{0}}^{t_{1}}\left\langle\zeta(t), u^{\prime}(t)\right\rangle d t+M\left\|u^{\prime}-u\right\|_{2}^{2} \\
\geq \int_{t_{0}}^{t_{1}} G(t, x(t), u(t), v(t)) d t+g\left(x\left(t_{1}\right)\right)-\int_{t_{0}}^{t_{1}}\langle\zeta(t), u(t)\rangle d t .
\end{gathered}
$$

Hence $(x, u, v)$ is a solution of the following optimal control problem:

$$
\begin{array}{ll}
\min & \int_{t_{0}}^{t_{1}}\left[G\left(t, x^{\prime}(t), u^{\prime}(t), v^{\prime}(t)\right)-\left\langle\zeta(t), u^{\prime}(t)\right\rangle\right] d t+g\left(x^{\prime}\left(t_{1}\right)\right) \\
\text { s.t. } & \dot{x}^{\prime}(t)=\phi\left(t, x^{\prime}(t), u^{\prime}(t), v^{\prime}(t)\right) \quad \text { a.e. } \\
& x^{\prime}\left(t_{0}\right)=x_{0} \\
& v^{\prime}(t) \in V(t) \quad \text { a.e. } \\
& u^{\prime}(t) \in U(t):=\left\{u^{\prime} \in R^{n}:\left|u^{\prime}-u(t)\right| \leq \epsilon\right\} .
\end{array}
$$

In the proof of Theorem 5.2.1. of Clarke [3], if we replace the the Clarke generalized gradient $\partial$ by the limiting subgradient $\hat{\partial}$ in the transversality conditions, the argument goes through without modification [cf. Clarke [4]]. Applying Theorem 5.2.1. of Clarke [3] with the Clarke generalized gradient replaced by the limiting subgradient in the transversality conditions to the above optimal control problem with free end points leads to the existence of an1 arc $p_{2}$ such that

$$
\begin{aligned}
& -\dot{p_{2}}(t)=\nabla_{x} \phi(t, x(t), u(t), v(t))^{\top} p_{2}(t)-\nabla_{x} G(t, x(t), u(t), v(t)) \\
& \max _{u \in R^{n}, v \in V(t)}\left\{p_{2}(t) \cdot \phi(t, x(t), u, v)-G(t, x(t), u, v)-\langle\zeta(t), u\rangle\right\}
\end{aligned}
$$




$$
\begin{aligned}
& =p_{2}(t) \cdot \phi(t, x(t), u(t), v(t))-G(t, x(t), u(t), v(t))-\langle\zeta(t), u(t)\rangle \text { a.e. } \\
& -p_{2}\left(t_{1}\right) \in \hat{\partial} g\left(x\left(t_{1}\right)\right)
\end{aligned}
$$

where ${ }^{\top}$ denotes the transpose. (3) implies

$$
\begin{aligned}
\max _{v \in V(t)} & \left\{p_{2}(t) \cdot \phi(t, x(t), u(t), v)-G(t, x(t), u(t), v)\right\} \\
\quad= & p_{2}(t) \cdot \phi(t, x(t), u(t), v(t))-G(t, x(t), u(t), v(t)) \text { a.e. }
\end{aligned}
$$

and

$$
\zeta(t)=\nabla_{u} \phi(t, x(t), u(t), v(t))^{\top} p_{2}(t)-\nabla_{u} G(t, x(t), u(t), v(t)) .
$$

Step 2. For any $\zeta \in \hat{\partial} V(u)$, by definition, $\zeta=$ weak $^{*} \lim _{i \rightarrow \infty} \zeta_{i}$ where $\zeta_{i} \in \partial^{\pi} V\left(u_{i}\right)$, $u_{i} \rightarrow u$ in $L^{2}$ and $V\left(u_{i}\right) \rightarrow V(u)$. By step 1 , for each $u_{i}$, there eixsts an arc $p_{2}^{i}$ and an $\operatorname{arc} x_{i}$ which solves $P_{2}\left(u_{i}\right)$ (along with $v_{i}$ ) such that

$$
\begin{aligned}
& -\dot{p}_{2}^{i}(t)=\nabla_{x} \phi\left(t, x_{i}(t), u_{i}(t), v_{i}(t)\right)^{\top} p_{2}^{i}(t)-\nabla_{x} G\left(t, x_{i}(t), u_{i}(t), v_{i}(t)\right) \text { a.e. } \\
& \max _{v \in V(t)}\left\{p_{2}^{i}(t) \cdot \phi\left(t, x_{i}(t), u_{i}(t), v\right)-G\left(t, x_{i}(t), u_{i}(t), v\right)\right\} \\
& \quad=p_{2}^{i}(t) \cdot \phi\left(t, x_{i}(t), u_{i}(t), v_{i}(t)\right)-G\left(t, x_{i}(t), u_{i}(t), v_{i}(t)\right) \text { a.e. } \\
& \zeta_{i}(t)=\nabla_{u} \phi\left(t, x_{i}(t), u_{i}(t), v_{i}(t)\right)^{\top} p_{2}^{i}(t)-\nabla_{u} G\left(t, x_{i}(t), u_{i}(t), v_{i}(t)\right) \\
& -p_{2}^{i}\left(t_{1}\right) \in \hat{\partial} g\left(x_{i}\left(t_{1}\right)\right) .
\end{aligned}
$$

By [Theorem 2.8.2, Clarke [3]], (7) (8) and (9) implies that

$$
\begin{aligned}
& \left(-{\dot{p_{2}}}^{i}(t), \zeta_{i}(t), \dot{x_{i}}(t)\right) \in \partial H_{2}\left(t, x_{i}(t), u_{i}(t), p_{2}^{i}(t)\right) \quad \text { a.e. } \\
& -p_{2}^{i}\left(t_{1}\right) \in \hat{\partial} g\left(x_{i}\left(t_{1}\right)\right)
\end{aligned}
$$

From (7), one has

$$
\left|{\dot{p_{2}}}^{i}(t)\right| \leq k(t)\left(\left|p_{2}^{i}(t)\right|+1\right)
$$

By assumption (A8), (9) implies that

$$
\begin{aligned}
\left|p_{2}^{i}(t)\right| & \leq\left|\left(\nabla_{u} \phi\right)^{-1}\right|\left|\zeta_{i}(t)\right|+\left|\left(\nabla_{u} \phi\right)^{-1}\right|\left|\nabla_{u} G\right| \\
& \leq M\left(\left|\zeta_{i}(t)\right|+\left|\nabla_{u} G\right|\right)
\end{aligned}
$$

which implies that $\left\|p_{2}^{i}\right\|_{2}$ is bounded since $\left\|\zeta_{i}\right\|_{2}$ is bounded and $\left|\nabla_{u} G\right|$ is bounded by a $L^{2}$ function. Therefore $p_{2}^{i}(0)$ admit a uniform bound and (12) implies that $\left\|\dot{p}_{2}{ }^{i}\right\|_{1}$ is 
bounded by Hölder's inequality. Hence $\left\|p_{2}^{i}-p_{2}^{i}(0)\right\|_{\infty}$ is bounded which implies that $\left\|p_{2}^{i}\right\|_{\infty}$ is bounded. It follows that the set-valued map $\partial H_{2}$ is integrably bounded. Applying Proposition 2.1 to differential inclusion (10) with boundary conditon (11), one concludes that there exist a convergent subsequence of $\left\{x_{i}, p_{2}^{i}\right\}$ which converges to the arcs $x, p_{2}$ such that

$$
\left(-\dot{p_{2}}(t), \zeta(t), \dot{x}(t)\right) \in \partial H_{2}\left(t, x(t), u(t), p_{2}(t)\right) \quad \text { a.e. }
$$

Note that by Lemma 2.1 we may suppose $x \in Y_{u}$ since $x_{i}$ is an optimal trajectory of $P_{2}\left(u_{i}\right)$. From the semicontinuity of the limiting subgradients, one also have

$$
-p_{2}\left(t_{1}\right) \in \hat{\partial} g\left(x\left(t_{1}\right)\right) \text {. }
$$

Therefore we conclude that

$\hat{\partial} V(u) \subset \cup_{x \in Y_{u}}\left\{\zeta: \exists \operatorname{arc} p_{2}\right.$ s.t. $\left(-\dot{p_{2}}, \zeta, \dot{x}\right) \in \partial H_{2}\left(t, x, u, p_{2}\right)$ a.e., $\left.-p_{2}\left(t_{1}\right) \in \hat{\partial} g\left(x\left(t_{1}\right)\right)\right\}$.

Step 3. To complete the proof of the theorem, one only have to show that $V$ is Lipschitz near $u$. By a recent result of Clarke et al [Theorem 3.6. of [7]], $V$ is Lipschitz near $u$ of rank $C$ if and only if

$$
\sup \left\{\|\zeta\|_{2}: \zeta \in \partial^{\pi} V\left(u^{\prime}\right)\right\} \leq C \quad \forall u^{\prime} \text { in a neighorhood of } u .
$$

Indeed, by Step 1 , for any $u$ and any $\zeta \in \partial^{\pi} V(u)$ there exists an arc $p_{2}$ along with a solution $(x, v)$ of $P_{2}(u)$ such that (2), (4) and (6) hold. Therefore one has

$$
\begin{aligned}
& \left|\dot{p_{2}}(t)\right| \leq k(t)\left(\left|p_{2}(t)\right|+1\right) \\
& \left|p_{2}\left(t_{1}\right)\right| \leq K_{g} \\
& |\zeta(t)| \leq M\left(\left|p_{2}(t)\right|+\left|\nabla_{u} G\right|\right) .
\end{aligned}
$$

Together with Gronwall's lemma, (13) and (14) yield an a priori bound on all such $\left\|p_{2}\right\|_{\infty}$. It then follows from (15) that all $\zeta \in \partial^{\pi} V(u), \forall u \in L^{2}\left(\left[t_{0}, t_{1}\right], R^{n}\right)$ are bounded in $L^{2}$. Hence $V$ is Lipschitz continuous. and the proof of Theorem 3.1 is now complete.

\section{$3 \quad$ Necessary conditions for optimality}

In this section we derive the necessary optimality condition for the nonstandard problem with a functional constraint. Our approach is to reformulate the original problem 
as an infinite dimensional optimization problem and derive the desired result from the necessary optimality condition for this infinite dimensional optimization problem. Formulation as an infinite dimensional optimization problem takes care the functional constraints. However, usual Lagrange multiplier rule for infinite dimensional optimization problems can not be used here since problem data are not Lipschitz in the control variable in the lower level optimal control problem. Ioffe [8] derived a very general maximum principle for the standard optimal control problem by reduction to an infinite dimensional optimization problem. We will use the result and approach of Ioffe to derive the necessary optimality condition of maxmum principle type for the bilevel dynamic problem.

Define the pseudo Hamiltonian for problem $\left(\tilde{P}_{1}\right)$ as

$$
H_{1}\left(t, x, u, v, p_{1} ; \lambda, r\right):=p_{1} \cdot \phi(t, x, u, v)-r G(t, x, u, v)-\lambda F(t, x, u, v),
$$

for $t \in\left[t_{0}, t_{1}\right], x, p_{1} \in \mathbb{R}^{d}, u \in R^{n}, v \in R^{m}, \lambda, r \in \mathbb{R}$.

Theorem 3.1 Assume that assumptions (A1)-(A4) hold. Let $\left(u^{*}(t), v^{*}(t)\right)$ be an optimal strategy of the bilevel dynamic problem $P_{1}$ and $x^{*}(t)$ the corresponding trajectory. Assume that the value function for the lower level problem $V$ is locally Lipschitz continuous near $u^{*}$. Then there exist $\lambda \geq 0, r \geq 0$ and an arc $p_{1}$ such that:

$$
\begin{aligned}
& -\dot{p_{1}}(t)=\nabla_{x} H_{1}\left(t, x^{*}(t), u^{*}(t), v^{*}(t) ; \lambda, r\right) \\
& \max _{v \in V(t)} H_{1}\left(t, x^{*}(t), u^{*}(t), v, p_{1}(t) ; \lambda, r\right)=H_{1}\left(t, x^{*}(t), u^{*}(t), v^{*}(t), p_{1}(t) ; \lambda, r\right) \\
& -p_{1}\left(t_{1}\right) \in \lambda \partial f\left(x^{*}\left(t_{1}\right)\right)+r \partial g\left(x^{*}\left(t_{1}\right)\right) \\
& \nabla_{u} H_{1}\left(t, x^{*}(t), u^{*}(t), v^{*}(t) ; \lambda, r\right) \in r \partial V\left(u^{*}\right) \\
& \left\|p_{1}\right\|_{\infty}+\lambda+r>0,
\end{aligned}
$$

where $\|\cdot\|_{\infty}$ denotes the supremum norm.

Proof. We pose the optimal control problem $\widetilde{P}_{1}$ equivalently as the following problem

$$
\begin{aligned}
& \widehat{P}_{1} \quad \min f\left(x\left(t_{1}\right)\right)+z\left(t_{1}\right) \\
& \text { s.t. } \quad \dot{x}(t)=\phi(t, x(t), u(t), v(t)) \quad \text { a.e. } \\
& \dot{y}(t)=G(t, x(t), u(t), v(t)) \quad \text { a.e. } \\
& \dot{z}(t)=F(t, x(t), u(t), v(t)) \quad \text { a.e. }
\end{aligned}
$$




$$
\begin{aligned}
& v(t) \in V(t) \quad \text { a.e. } \\
& (x, y, z)\left(t_{0}\right) \in\left\{x_{0}\right\} \times\{0\} \times\{0\} \\
& V(u) \geq y\left(t_{1}\right)+g\left(x\left(t_{1}\right)\right) .
\end{aligned}
$$

We now reformulate the above problem as an infinite dimensional optimization problem. Let $C\left(\left[t_{0}, t_{1}\right], R^{n}\right)$ be the space of continuous mappings from $\left[t_{0}, t_{1}\right]$ into $R^{n}$ with the usual supremum norm. Let $\mathcal{V}$ denote the collection of all admissible control functions for $P_{2}(u)$. Set

$$
\tilde{x}:=(x, y, z), \quad \tilde{\phi}:=(\phi, G, F)
$$

For $v(\cdot) \in \mathcal{V}$, the mapping $(\tilde{x}(\cdot), u(\cdot)) \rightarrow F_{0}(\widetilde{x}(\cdot), u(\cdot), v(\cdot))$ from $X:=C\left(\left[t_{0}, t_{1}\right], R^{d+2}\right) \times$ $L^{2}\left(\left[t_{0}, t_{1}\right], R^{n}\right)$ into $Y:=C\left(\left[t_{0}, t_{1}\right], R^{d+2}\right)$ :

$$
F_{0}(\widetilde{x}(\cdot), u(\cdot), v(\cdot))(t):=\widetilde{x}(t)-\widetilde{x}\left(t_{0}\right)+\int_{t_{0}}^{t} \tilde{\phi}(s, \widetilde{x}(s), u(s), v(s)) d s
$$

is well defined and continuously (Gâteaux) differentiable in $(\tilde{x}(\cdot), u(\cdot))$. Let finally

$$
\begin{aligned}
& f_{0}(\tilde{x}(\cdot)):=f\left(x\left(t_{1}\right)\right)+z\left(t_{1}\right) \\
& G_{0}(\tilde{x}(\cdot), u(\cdot)):=y\left(t_{1}\right)+g\left(x\left(t_{1}\right)\right)-V(u) \\
& S:=\left\{\tilde{x} \subset Y: x\left(t_{0}\right)=x_{0}, y\left(t_{0}\right)=0, z\left(t_{0}\right)=0\right\} .
\end{aligned}
$$

Then Problem $\hat{P}_{1}$ is equivalent to the problem

$$
\begin{array}{ll}
P_{1}^{\prime} \quad \min & f_{0}(\widetilde{x}) \\
\text { s.t. } & F_{0}(\tilde{x}, u, v)=0 \\
& G_{0}(\widetilde{x}, u) \leq 0 \\
& (\tilde{x}, u) \in S \times L^{2} \\
& v \in \mathcal{V} .
\end{array}
$$

The above problem is in the form of a very general problem in $\S 4$ of Ioffe [8]. Let the Lagrangian of the above problem be

$$
L(\lambda, r, \alpha, \widetilde{x}, u, v):=\lambda f_{0}(\tilde{x})+r G_{0}(\widetilde{x}, u)+\left\langle\alpha, F_{0}(\widetilde{x}, u, v)\right\rangle
$$

As in $\S 5$ of Ioffe [8], one can verify that the assumptions for Theorem 2 of Ioffe [8] are satisfied. By Theorem 2 of Ioffe [8], if $\left(x^{*}, u^{*}, v^{*}\right)$ is a local solution to $P_{1}^{\prime}$, then 
there exist Lagrange multipliers $\lambda \geq 0, r \geq 0, \alpha \in Y^{*}$ not all equal to zero such that

$$
\begin{aligned}
& 0 \in \partial_{(\widetilde{x}, u)} L\left(\lambda, r, \alpha, \widetilde{x}^{*}, u^{*}, v^{*}\right)+N_{S}\left(\widetilde{x}^{*}\right) \times\{0\} \\
& L\left(\lambda, r, \alpha, \widetilde{x}^{*}, u^{*}, v^{*}\right)=\min _{v \in \mathcal{V}} L\left(\lambda, r, \alpha, \widetilde{x}^{*}, u^{*}, v\right) \\
& r G_{0}\left(\widetilde{x}^{*}, u^{*}\right)=0,
\end{aligned}
$$

where $Y^{*}$ denotes the space of continuous linear functions on $Y$. (21) implies that

$$
\begin{aligned}
& 0 \in \partial_{\tilde{x}} L\left(\lambda, r, \alpha, \widetilde{x}^{*}, u^{*}, v^{*}\right)+N_{S}\left(\widetilde{x}^{*}\right) \\
& \subset \lambda \partial f_{0}\left(\widetilde{x}^{*}\right)+r \partial_{\tilde{x}} G_{0}\left(\widetilde{x}^{*}, u^{*}\right)+D_{\tilde{x}}\left\langle\alpha, F_{0}\left(\widetilde{x}^{*}, u^{*}, v^{*}\right)\right\rangle+N_{S}\left(\widetilde{x}^{*}\right) \\
& 0 \in r \partial_{u} G_{0}\left(\widetilde{x}^{*}, u^{*}\right)+D_{u}\left\langle\alpha, F_{0}\left(\widetilde{x}^{*}, u^{*}, v^{*}\right)\right\rangle \\
& =-r \partial V\left(u^{*}\right)+D_{u}\left\langle\alpha, F_{0}\left(\widetilde{x}^{*}, u^{*}, v^{*}\right)\right\rangle \text {. }
\end{aligned}
$$

Notice that $\left\langle\alpha, F_{0}(\widetilde{x}, u, v)\right\rangle$ can be represented as

$$
\begin{aligned}
& \left\langle\alpha, F_{0}(\widetilde{x}, u, v)\right\rangle \\
& \quad=\int_{t_{0}}^{t_{1}}\left\langle\widetilde{x}(s)-\widetilde{x}\left(t_{0}\right), \xi(s)\right\rangle d \mu-\int_{t_{0}}^{t_{1}}\left\langle\int_{t}^{t_{1}} \xi(\tau) d \mu, \widetilde{\phi}(t, \tilde{x}(t), u(t), v(t))\right\rangle d t
\end{aligned}
$$

where the pair $(\mu, \xi(\cdot))$ represents the functional $\alpha \in Y^{*}$ ( $\mu$ being a nonnegative Radon measure on $\left[t_{0}, t_{1}\right]$ and $\xi(\cdot):\left[t_{0}, t_{1}\right] \rightarrow R^{d+2}, \mu$-integrable), i.e.,

$$
\int_{t_{0}}^{t_{1}}\langle\xi(t), y(t)\rangle d \mu=\langle\alpha, y(\cdot)\rangle, \quad \forall y(\cdot) \in Y_{0}
$$

Now let us analyse (24). We have: $\partial f(\widetilde{x}(\cdot))$ contains those $\beta \in Y^{*}$ which can be represented in the form

$$
\langle\beta, h(\cdot)\rangle=\left\langle a, h\left(t_{1}\right)\right\rangle
$$

for some $a \in \partial f\left(x\left(t_{1}\right)\right) \times\{0\} \times\{1\}$.

Similarly, $\partial_{\widetilde{x}} G_{0}(\widetilde{x}, u)$ contains those $\beta \in Y^{*}$ which can be represented in the form

$$
\langle\beta, h(\cdot)\rangle=\left\langle b, h\left(t_{1}\right)\right\rangle
$$

for some $b \in \partial g\left(x\left(t_{1}\right)\right) \times\{1\} \times\{0\}$.

Let $p(t):=\int_{t}^{t_{1}} \xi(\tau) d \mu$. Then $p$ is an arc. Let $D_{\widetilde{x}}\left\langle\alpha, F_{0}(\widetilde{x}, u, v)\right\rangle$ denote the Gâteaux derivative of the functional $\left\langle\alpha, F_{0}(\widetilde{x}, u, v)\right\rangle$ with respect to $\widetilde{x}$. Then for any $h \in X$, one has

$$
\begin{aligned}
\left\langle D_{\widetilde{x}}\left\langle\alpha, F_{0}(\widetilde{x}, u, v)\right\rangle, h(\cdot)\right\rangle= & \int_{t_{0}}^{t_{1}}\left\langle h(t)-h\left(t_{0}\right), \xi(t)\right\rangle d \mu \\
& +\int_{t_{0}}^{t_{1}}\left\langle\nabla_{\widetilde{x}} \widetilde{\phi}(t, \tilde{x}(t), u(t), v(t))^{\top} p(t), h(t)\right\rangle d t
\end{aligned}
$$


$N_{S}(\widetilde{x})$ contains those $\beta \in Y^{*}$ which can be represented in the form

$$
\langle\beta, h(\cdot)\rangle=\left\langle c, h\left(t_{0}\right)\right\rangle
$$

for some $c \in N_{\left\{x_{0}\right\} \times\{0\} \times\{0\}}\left(\widetilde{x}\left(t_{0}\right)\right)$.

(24) yields the existence of

$a \in \partial f\left(x^{*}\left(t_{1}\right)\right) \times\{0\} \times\{1\}, b \in \partial g\left(x^{*}\left(t_{1}\right)\right) \times\{1\} \times\{0\}, c \in N_{\left\{x_{0}\right\} \times\{0\} \times\{0\}}\left(\widetilde{x}^{*}\left(t_{0}\right)\right)$

such that

$$
\begin{aligned}
0= & \lambda\left\langle a, h\left(t_{1}\right)\right\rangle+r\left\langle b, h\left(t_{1}\right)\right\rangle+\int_{t_{0}}^{t_{1}}\left\langle h(t)-h\left(t_{0}\right), \xi(t)\right\rangle d \mu \\
& +\int_{t_{0}}^{t_{1}}\left\langle\nabla_{\widetilde{x}} \tilde{\phi}\left(t, \widetilde{x}^{*}(t), u^{*}(t), v^{*}(t)\right)^{\top} p(t), h(t)\right\rangle d t+\left\langle c, h\left(t_{0}\right)\right\rangle \quad \forall h \in X .
\end{aligned}
$$

Let us denote $h=\left(h_{1}, h_{2}, h_{3}\right), \xi=\left(\xi_{1}, \xi_{2}, \xi_{3}\right), p=\left(p_{1}, p_{2}, p_{3}\right)$ where 1 corresponding vectors in $R^{d}, 2,3$ to those in $R$. Especially, if we choose $h(\cdot)$ which are absolutely continuous and $h\left(t_{0}\right)=0, h_{i}\left(t_{1}\right)=0$ for $i=1,3$, we have

$$
0=r h_{2}\left(t_{1}\right)+\int_{t_{0}}^{t_{1}} h_{2}(t) \xi_{2}(t) d \mu
$$

which is equal to

$$
0=\int_{t_{0}}^{t_{1}}\left(\int_{t}^{t_{1}} \xi_{2}(s) d \mu+r\right) d h_{2}(t)
$$

which implies that $p_{2}(t)=-r$.

Similarly, if we choose $h(\cdot)$ which are absolutely continuous and $h\left(t_{0}\right)=0, h_{i}\left(t_{1}\right)=$ 0 for $i=1,2$, we have

$$
0=\lambda h_{3}\left(t_{1}\right)+\int_{t_{0}}^{t_{1}} h_{3}(t) \xi_{3}(t) d \mu
$$

which implies that $p_{3}(t)=-\lambda$.

If we choose $h(\cdot)$ which are absolutely continuous and $h\left(t_{0}\right)=0, h_{i}\left(t_{1}\right)=0$ for $i=2,3$, we have

$$
\begin{aligned}
0= & \lambda\left\langle a, h_{1}\left(t_{1}\right)\right\rangle+r\left\langle b_{1}, h_{1}\left(t_{1}\right)\right\rangle+\int_{t_{0}}^{t_{1}}\left\langle h_{1}(t), \xi_{1}(t)\right\rangle d t t \\
& +\int_{t_{0}}^{t_{1}}\left\langle\nabla_{x} \tilde{\phi}\left(t, \tilde{x}^{*}(t), u^{*}(t), v^{*}(t)\right)^{\top} p(t), h_{1}(t)\right\rangle d t
\end{aligned}
$$

Setting $-q=\lambda a_{1}+r b_{1}$ and changing the order of integration, we obtain

$$
\begin{aligned}
0= & \int_{t_{0}}^{t_{1}}\left\langle\int_{t}^{t_{1}} \xi_{1}(t) d \mu+\nabla_{x} \phi\left(t, x^{*}(t), u^{*}(t), v^{*}(t)\right)^{\top} p_{1}(t)\right. \\
& \left.-r \nabla_{x} G\left(t, x^{*}(t), u^{*}(t), v^{*}(t)\right)-\lambda \nabla_{x} F\left(t, x^{*}(t), u^{*}(t), v^{*}(t)\right)-q, k(t)\right\rangle d t
\end{aligned}
$$


where $k(t)=\dot{h}(t)$ is an arbitrary integrable mapping. In view of definition of $p_{1}(t)$, this implies

$$
\begin{aligned}
p_{1}(t)-q= & \int_{t}^{t_{1}}\left(\nabla_{x} \phi\left(s, x^{*}(s), u^{*}(s), v^{*}(s)\right)^{\top} p_{1}(s)\right. \\
& \left.-r \nabla_{x} G\left(s, x^{*}(s), u^{*}(s), v^{*}(s)\right)-\lambda \nabla_{x} F\left(s, x^{*}(s), u^{*}(s), v^{*}(s)\right)\right) d s .
\end{aligned}
$$

from which we derive (16).

(19) are derived from (25) in an exactly similar way.

We also have

$$
p\left(t_{1}\right)=q \in-\lambda \partial f\left(x^{*}\left(t_{1}\right)\right)-r \partial g\left(x^{*}\left(t_{1}\right)\right)
$$

That is (18).

(22) implies that

$$
-\int_{t_{0}}^{t_{1}}\left\langle p(t), \widetilde{\phi}\left(t, x^{*}(t), u^{*}(t), v^{*}(t)\right)\right\rangle d t \leq-\int_{t_{0}}^{t_{1}}\left\langle p(t), \tilde{\phi}\left(t, x^{*}(t), u^{*}(t), v(t)\right)\right\rangle d t .
$$

Since $-\lambda=p_{2}(t), r=-p_{3}(t)$, the above inequality implies that

$$
\int_{t_{0}}^{t_{1}} H_{1}\left(t, x^{*}(t), u^{*}(t), v^{*}(t), p_{1}(t) ; \lambda, r\right) d t \geq \int_{t_{0}}^{t_{1}} H_{1}\left(t, x^{*}(t), u^{*}(t), v(t), p_{1}(t) ; \lambda, r\right) d t
$$

for any $v(\cdot) \in \mathcal{V}$. Since for any measurable set $E \subset\left[t_{0}, t_{1}\right]$, one has

$$
v(\cdot)=\chi_{E}(\cdot) v(\cdot)+\left(1-\chi_{E}(\cdot)\right) v^{*}(\cdot)
$$

belongs to $\mathcal{V}$ whenever $v(\cdot) \in \mathcal{V}$, it follows that

$$
H_{1}\left(t, x^{*}(t), u^{*}(t), v^{*}(t), p_{1}(t) ; \lambda, r\right) \geq H_{1}\left(t, x^{*}(t), u^{*}(t), v(t), p_{1}(t) ; \lambda, r\right) \text { a.e. } t
$$

for any $v(\cdot) \in \mathcal{V}$. From measurable selection theory, (17) follows.

(20) follows easily from the fact that $\lambda, r, \alpha$ are not all equal to zero. The proof of the theorem is now complete.

Combining Theorem 3.1 and Theorem 2.1, one has the following necessary conditions for optimality for the general bilevel dynamic problem.

Theorem 3.2 Assume assumptions (A1)-(A8) hold. Let $\left(u^{*}(t), v^{*}(t)\right)$ be an optimal strategy of the bilevel dynamic problem $P_{1}$ and $x^{*}(t)$ the corresponding trajectory. Then there exist $\lambda \geq 0, r \geq 0$ and arc $p_{1}$ such that:

$$
\begin{aligned}
& -\dot{p_{1}}(t)=\nabla_{x} H_{1}\left(t, x^{*}(t), u^{*}(t), v^{*}(t) ; \lambda, r\right) \\
& \max _{v \in V(t)} H_{1}\left(t, x^{*}(t), u^{*}(t), v, p_{1}(t) ; \lambda, r\right)=H_{1}\left(t, x^{*}(t), u^{*}(t), v^{*}(t), p_{1}(t) ; \lambda, r\right) \quad \text { a.e. }
\end{aligned}
$$




$$
\begin{aligned}
& -p_{1}\left(t_{1}\right) \in \lambda \partial f\left(x^{*}\left(t_{1}\right)\right)+r \partial g\left(x^{*}\left(t_{1}\right)\right) \\
& \nabla_{u} H_{1}\left(t, x^{*}(t), u^{*}(t), v^{*}(t) ; \lambda, r\right) \in r c l c o \cup_{x \in Y_{u}},\left\{\zeta: \exists \text { arc } p_{2}\right. \text { s.t. } \\
& \left.\quad\left(-\dot{p_{2}}, \zeta, \dot{x}\right) \in \partial H_{2}\left(t, x, u^{*}, p_{2}\right) \quad \text { a.e., } \quad-p_{2}\left(t_{1}\right) \in \hat{\partial} g\left(x\left(t_{1}\right)\right)\right\}, \\
& \left\|p_{1}\right\|_{\infty}+\lambda+r>0 .
\end{aligned}
$$

\section{Conclusion and future research}

In this paper we derive a necessary optimality condition for the bilevel dynamic problem. Due to the technical difficulties in studying the value function of optimal control problem with respect to general infinite-dimensional perturbations in dynamic and objective functions, we can only studied the problem without endpoint constraints. The perturbation structure we allowed are also limited. This will be subject to future research.

\section{References}

[1] J.F. Bard and J.E. Falk, An explicit solution to the multi-level programming problem, Oper. Res. 9(1982), 77-100.

[2] C.I. Chen and J.B. Cruz Jr., Stackelberg solution for two-person games with baised information patterns, IEEE trans. Auto. Control, 6(1972), 791-798.

[3] F.H. Clarke, Optimization and Nonsmooth Analysis, Wiley-Interscience, New York, 1983.

[4] F.H. Clarke, Methods of Dynamic and Nonsmooth Optimization, NSF-CBMS Regional Conference Series in Applied Mathematics, Society for Industrial and Applied Mathematics, Philadelphia, vol 57, 1989.

[5] F.H. Clarke, Perturbed optimal control problems, IEEE Transactions on Automatic Control, 6(1986), 535-542.

[6] F.H. Clarke and P.D. Loewen, The value function in optimal control: sensitivity, controllability, and time-optimality, SIAM J. Control and Optimization, 24(1986), 243-263. 
[7] F.H. Clarke, R.J. Stern and P.R. Wolenski, Subgradient criteria for monotonicity and the Lipschitz condition, Preprint, 1992.

[8] A.D. Ioffe, Necessary conditions in nonsmooth optimization, Mathematics of Operations Research, 9(1984), 159-189.

[9] R.T. Rockafellar, Extensions of subgradient calculus with applications to optimization, Nonlinear Analysis, Theory, Methods and Applications, 9(1985), 665698.

[10] H. von Stackelberg, The Theory of the Market Economy, Oxford University Press, Oxford, 1952.

[11] J.J. Ye, Necessary conditions for bilevel dynamic optimization problems, Submitted for publication, 1993.

[12] J.J. Ye and D.L. Zhu, Optimality conditions for bilevel programming problems, Submitted for publication.

[13] Roxin Zhang, Problems of Hierarchical Optimization: Nonsmoothness and Analysis of Solutions, University of Washington, Ph.D. Thesis, 1990.

[14] Roxin Zhang, Problems of hierarchical optimization in finite dimensions, To appear in SIAM J. Optimization. 